\title{
THE MONOTONE UNION PROPERTY OF MANIFOLDS
}

\author{
BY
}

\section{LAWRENCE S. HUSCH( $\left.{ }^{1}\right)$}

1. Introduction. Let $M$ be a manifold. $U=\bigcup_{i=1}^{\infty} U_{i}$ is called a monotone union of $M$ if (i) each $U_{i}$ is homeomorphic to $M$ and (ii) $U_{i} \subset$ int $U_{i+1}$ (=interior $U_{i+1}$ ) for each $i$. $M$ is said to have the monotone union property if each monotone union of $M$ is homeomorphic to the interior of $M$. Brown [5] has shown that the $n$-cell has the monotone union property. Kwun [13] has generalized this to all compact combinatorial $n$-manifolds $(n \neq 4)$ with a sphere boundary. Edwards [7, p. 9], $[8$, p. 421] has given some necessary and sufficient conditions that a monotone union of a compact 3-manifold be homeomorphic to int $M$. By use of the Regular Neighborhood Annulus Theorem [11, p. 725], Edwards' results may be generalized to compact $n$-manifolds for all $n$.

The motivation for this paper was the converse of Kwun's Theorem.

(A) If $M$ is a compact bounded combinatorial manifold with the monotone union property, is the boundary of $M$ a sphere?

We shall restrict ourselves to connected compact 3-manifolds whose boundary is either a collection of spheres or contains a torus, i.e., a surface of genus 1. A positive answer is given for (A) for a class of such manifolds in $\S 4$ and $\S 5$. However, the answer to (A) is no; an example of a compact bounded 3-manifold whose boundary is a torus and which possesses the monotone union property is constructed in $\S 6$.

Whitehead [25] has given an example of a monotone union of the solid torus which is not homeomorphic to the interior of any compact bounded manifold. However, there exist manifolds which do not have the monotone union property but they have the property that each monotone union is homeomorphic to the interior of some compact bounded manifold. Manifolds with the latter property are said to have the bounded union property. A characterization of a class of manifolds with the bounded union property is given in $\S 4$ and $\S 5$.

The author expresses his gratitude to the topologists at Florida State University for their patience and help in the discussions on this paper and especially to Dr. S. Kinoshita for his invaluable suggestions leading to the proofs of Theorem 4 and Lemma 6.

2. Whitehead's example. Let us recall Whitehead's construction in [25]. Let

Presented to the Society, November 11, 1966; received by the editors February 1, 1967.

(1) The results appearing in this paper form a part of the author's dissertation submitted as a partial requirement of the Ph.D. degree at Florida State University under the direction of Professor J. J. Andrews. Research was supported in part by NSF Grant GP-5458. 
$T_{2}$ be an unknotted polyhedral solid torus in the three sphere $S^{3}$. Let $T_{1}$ be a polyhedral solid torus embedded in the interior of $T_{2}$ as indicated in Figure 1. Let $B$ be a polyhedral 3-cell in the interior of $T_{1}$ as indicated. Clearly we can find a homeomorphism $h$ of $S^{3}$ onto itself such that

(i) $h(x)=x$ for $x \in B$;

(ii) $h\left(T_{1}\right)=T_{2}$.

Let $U=\bigcup_{i=1}^{\infty} h^{n}\left(T_{1}\right)$. Then $U$ is a monotone union of the solid torus $T_{1}$. Whitehead showed that $U$ was contractible, so that $T_{1}$ does not have the monotone union property.

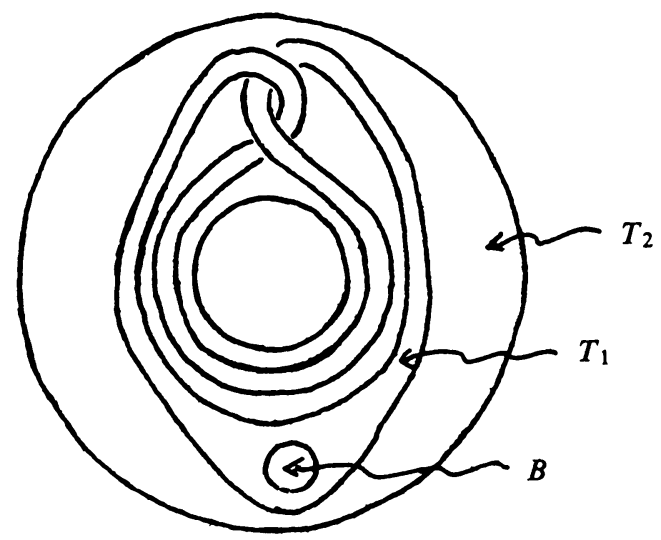

FIGURE 1

Proposition 1. $U$ is not homeomorphic to the interior of any compact bounded 3-manifold.

Proof. Suppose to the contrary that $U$ is homeomorphic to int $N$, where $N$ is a compact bounded 3-manifold. By [3], [16], we may assume that $N$ is a combinatorial manifold. Let $N_{1}=N-\operatorname{int} T_{1}$. By Alexander [2, Corollary 14:4], $N_{1}$ is a compact combinatorial manifold. Hence, the fundamental group of $N_{1}$ is finitely generated [10, Corollary 6.3.10]. However, Newman and Whitehead [18] have shown that the fundamental group of $N_{1}$ is infinitely generated. This contradiction establishes the proposition.

3. The concentricity property for 3-manifolds. Henceforth we shall assume, without loss of generality, that our manifolds are combinatorial and that all maps are piecewise linear. In [8], Edwards makes the following definition.

DefNition. Two compact manifolds $M$ and $N$ with nonempty boundaries and $N \subset$ int $M$ are said to be concentric if $\mathrm{Cl}(M-N)$ is homeomorphic to (bdry $M) \times I$, where $I$ is the closed interval $[0,1]$.

We make the following definition.

Definition. A compact manifold $M$ is said to have the concentricity property if for each embedding $h: M \rightarrow$ int $M, h(M)$ and $M$ are concentric. 
THEOREM 1. Let $M$ be a compact 3-manifold with connected boundary. $M$ has the monotone union property if and only if $M$ has the concentricity property.

Proof. If $M$ has the concentricity property, clearly $M$ has the monotone union property. Suppose that $M$ has the monotone union property and let $h: M \rightarrow$ int $M$ be an embedding. For each positive integer $j$, consider $M_{j}=M \times j$. Define $h_{j}: M_{j} \rightarrow M_{j+1}$ by $h_{j}(x, j)=(h(x), j+1) .\left\{M_{j}, h_{j}\right\}$ induces naturally a direct system $[23$, p. 18] in the category of topological spaces and continuous maps. The direct limit, $N$, of $\left\{M_{j}, h_{j}\right\}$ exists and can be constructed as in [23, p. 3] from $\bigcup M_{j}$. The natural maps $p_{i}: M_{i} \rightarrow N$ are continuous by definition and, in our case, are one-one. It is then easily seen that $N=\bigcup p_{i} M_{i}$ is a monotone union for $M$. Hence $N$ is homeomorphic to int $M$; let $k: N \rightarrow$ int $M$ be the homeomorphism. There exists a homeomorphism $c$ : (bdry $M) \times I \rightarrow M$ such that $c(x, 0)=x$. Hence $k^{-1} c$ : (bdry $\left.M\right) \times(0,1] \rightarrow N$ is a homeomorphism. There exists a positive integer $n$ such that $p_{n}($ bdry $M) \subset k^{-1} c[($ bdry $M) \times(0,1)]$. Since bdry $M$ is connected, $p_{n}($ bdry $M)$ separates $k^{-1} c($ bdry $M \times 1)$ from $k^{-1} c($ bdry $M \times \varepsilon)$ for some sufficiently small $\varepsilon$. From Edwards [8, Theorem 1] $p_{n}$ (bdry $M_{n}$ ) is concentric with $k^{-1} c$ (bdry $\left.M \times 1\right)$. Similarly, $p_{n+1}\left(\right.$ bdry $\left.M_{n+1}\right)$ is concentric with $k^{-1} c($ bdry $M \times 1)$. Hence, by [8, Theorem 2], $\mathrm{Cl}\left(p_{n+1} M_{n+1}-p_{n} M_{n}\right)$ is homeomorphic to (bdry $\left.M\right) \times I$. Therefore, it follows that $M$ has the concentricity property.

4. Manifolds whose boundary components are spheres. We shall prove the following theorem in this section.

THEOREM 2. Let $M$ be a compact orientable 3-manifold whose boundary components are spheres. Then

(i) $M$ has the bounded union property;

(ii) $M$ has the monotone union property if and only if the boundary of $M$ is connected.

Proposition 2. Let $M$ and $N$ be two compact 3-manifolds such that there exists embeddings $i: M \rightarrow$ int $N, j: N \rightarrow$ int $M$. If $M$ has the monotone union property and $M$ is not homeomorphic to $N$, then $N$ does not have the monotone union property.

Proof. Let $M_{i}$ be formed from $M \cup[($ bdry $M) \times[1,2-1 / i]]$ by identifying (bdry $M$ ) $\times 1$ with bdry $M$ by the map $y \times 1 \rightarrow y$ for each positive integer $i$. Hence $L=\bigcup M_{i}$ is a monotone union of $M$ and hence $L$ is homeomorphic to int $M$. By the hypothesis and Theorem 1, we can find for each positive integer $k$ an embedding $j_{k}: N \rightarrow L$ such that $j_{k}($ bdry $N) \subseteq[($ bdry $M) \times(2-1 /(k-1), 2-1 / k)]$. Clearly $L=\bigcup j_{k} N$ is then a monotone union for $N$. Since $M$ is not homeomorphic to $N$, int $M$ is not homeomorphic to int $N$ [8, Theorem 3]. Therefore $L$ is not homeomorphic to int $N$ and $N$ does not have the monotone union property.

Proposition 3. Let $M$ satisfy the hypotheses of Theorem 2; if the boundary of $M$ is not connected, then $M$ does not have the monotone union property. 
Proof. Let $n$ be the number of components of bdry $M$. Suppose bdry $M=\bigcup S_{i}$, where each $S_{i}$ is a 2-sphere. Let $\left\{B_{i}\right\}$ be a collection of $n$ distinct 3-cells. Let $M^{\prime}$ be obtained from $M \cup B_{1} \cup \cdots \cup B_{n}$ by identifying the boundary of each $B_{i}$ with $S_{i}$. Let $A_{1}, A_{2}, \ldots, A_{n}$ be arcs in $M$ such that

(i) $A_{i} \cap A_{j}=\varnothing$ for $i \neq j$;

(ii) $A_{i} \cap B_{j}$ is a point if $i=j-1$ or $j$;

(iii) $A_{i} \cap B_{j}=\varnothing$ otherwise.

Let $N$ be a regular neighborhood of $\left(\cup B_{i}\right) \cup\left(\cup A_{i}\right)$. By [11] $N$ is a 3-cell. Let $R=M^{\prime}$-int $N$. Then $R$ is a compact 3-manifold such that the boundary of $R$ is a 2-sphere. By Kwun [13], $R$ has the monotone union property. Let $D$ be a 3-cell contained in the interior of $B_{1}$. Let $R^{\prime}=M^{\prime}-$ int $D$. By Newman [17], $R$ and $R^{\prime}$ are homeomorphic. Therefore we have

$$
R \subset \text { int } M \subset M \subset \text { int } R^{\prime} .
$$

Since the boundary of $R$ is connected and the boundary of $M$ is not connected, $R$ and $M$ are not homeomorphic. By Proposition 2, $M$ does not have the monotone union property.

Proposition 4. Let $M$ satisfy the hypotheses of Theorem 2; then $M$ has the bounded union property.

Proof. We show that $M$ has the bounded union property by induction on the number of components of the boundary of $M$. If $k=1$, where $k$ is the number of components of the boundary of $M$, this is Kwun's Theorem. Suppose the theorem is true for all $k<n$. Let $k=n$ and let $U=\bigcup U_{i}$ be a monotone union of $M$. It is easy to see that the number of components of $U_{i+1}-U_{i}$ is $n$ for each $i$. We have two cases.

Case 1. Suppose there exists a number $N$ such that for every $i>N$, each component of $U_{i+1}-U_{i}$ meets the boundary of $U_{i+1}$. Let $A_{1}, A_{2}, \ldots, A_{n}$ be the components of $U_{i+1}$-int $U_{i}$. We want to show that each $A_{i}$ is homeomorphic to $S^{2} \times[0,1]$; then it would follow easily that $U$ is homeomorphic to int $M$. Let $M^{\prime}$ be the closed manifold obtained from $U_{i+1}$ by attaching $n$ disjoint 3-cells along the boundary of $U_{i+1}$; let $M^{\prime \prime}$ be obtained from $U_{i}$ similarly. Then $M^{\prime}$ and $M^{\prime \prime}$ are homeomorphic. Let $M_{i}$ be obtained from $A_{i}$ by attaching two 3-cells along the boundary of $A_{i}$. Then

$$
M^{\prime}=M^{\prime \prime} \# M_{1} \# M_{2} \# \cdots \# M_{n}
$$

( \# denotes the "connected sum" operation as defined in [15]. When we later form the connected sum of bounded manifolds, we shall assume as usual that the open 3-cells chosen shall have closures which do not meet their boundaries of the given manifolds.) By Milnor [15], $M_{1} \# M_{2} \# \cdots \# M_{n}$ is a 3-sphere and hence each $M_{i}$ is a 3-sphere. Therefore each $A_{i}$ is homeomorphic to $S^{2} \times[0,1]$.

Case 2. Suppose for each positive integer $N$, there exists an integer $i=i(N)>N$ 
such that some component of $U_{i+1}-U_{i}$ does not meet the boundary of $U_{i+1}$. Let $A_{1}, \ldots, A_{n}$ be the components of $U_{i+1}$-int $U_{i}$ so ordered that

(i) $A_{i} \cap$ bdry $U_{i+1} \neq \varnothing$ for $i=1,2, \ldots, r$;

(ii) $A_{i} \cap$ bdry $U_{i+1}=\varnothing$ for $i=r+1, \ldots, n$.

Each of the $A_{i}, i=r+1, \ldots, n$, have only one boundary component. Let $M^{\prime}, M^{\prime \prime}$, $M_{1}, M_{2}, \ldots, M_{r}$ be defined as in Case 1, except, of course, more than two 3-cells may have to be attached to each $A_{i}$. Form $M_{j}, j=r+1, \ldots, n$, by attaching a 3-cell to $A_{j}$. Then

$$
M^{\prime}=M^{\prime \prime} \# M_{1} \# M_{2} \# \cdots \# M_{n} .
$$

As in Case 1, each $M_{i}$ is a 3 -sphere so that the $A_{i}, i=r+1, \ldots, n$, are each 3-cells. Let $R$ be formed from $M$ by attaching a 3-cell along one of the boundary components of $M$. From the above remarks, it follows that there exists an embedding $h_{i}: R \rightarrow$ int $U_{i+1}$ such that

$$
U_{i} \subset \text { int } h_{i} R \subset h_{i} R \subset \text { int } U_{i+1} .
$$

Clearly, $U=\bigcup U_{i}=\bigcup_{N} h_{i(N)} R$ is a monotone union of $R$. By the induction hypotheses, $R$ has the bounded union property; therefore $U$ is homeomorphic to the interior of some compact bounded manifold. Hence $M$ has the bounded union property.

The proof of Theorem 2 now follows from Propositions 3 and 4. From the proof of Proposition 4, we also have:

COROLlaRY. Let $M$ satisfy the hypotheses of Theorem 2 and let $U=\bigcup U_{i}$ be a monotone union of $M$. Then $U$ is homeomorphic to the interior of the compact bounded manifold obtained from $M$ by attaching a collection (possibly empty) of 3-cells along some of the boundary components of $M$.

\section{Manifolds one of whose boundary components is a torus.}

THEOREM 3. Let $M$ be a compact 3-manifold whose boundary contains a torus $T$ which has the property that the inclusion map $i: T \rightarrow M$ induces $i_{\#}: \pi_{1}(T) \rightarrow \pi_{1}(M)$ which is either

(i) not a monomorphism; or

(ii) an epimorphism.

Then $M$ does not have the bounded union property; thus, in particular, $M$ does not have the monotone union property.

Proof. Suppose $i_{\#}$ is not a monomorphism. By the Loop Theorem [20], there is a simple closed polygonal curve $L$ on $T$ such that $L$ is essential in $T$ but inessential in $M$. By Dehn's Lemma [21], there is a polyhedral 2-cell $D$ in $M$ whose boundary is $L$. By using the collar of the boundary of $M$, we may assume that the interior of $D$ does not meet the boundary of $M$. Since $L$ is an essential simple closed curve in $T, L$ represents a generator of the fundamental group $\pi_{1}(T)$. Then we can attach a solid torus $R$ to $M$ by identifying the boundary of $R$ with $T$ so that $L$ and the core of 
$R$ bound a nonsingular annulus in $R$. Let $M_{\#}$ denote the resulting space and let $D_{\#}$ be the subset of $M_{\#}$ corresponding to $D \cup R$. Clearly $D_{\#}$ is a collapsible subcomplex of $M_{\#}$. Let $N$ be a regular neighborhood of $D_{\#}$ in $M_{\#}$ such that $N \cap($ bdry $M-T)$ $=\varnothing$. By [11], $N$ is a 3-cell. Using the notation of $\S 2$, let

$$
U_{i}=(M-\operatorname{int} N) \cup\left(\bigcup_{j=0}^{i} h^{j}\left(T_{1}\right)-\text { int } B\right)
$$

where the boundary of $N$ is identified with the boundary of $B$ for $i=0,1,2, \ldots$ Clearly each $U_{i}$ is homeomorphic to $M$ and $U_{i} \subset$ int $U_{i+1}$. Hence $U^{\prime}=\bigcup U_{i}$ $=M \cup U$ (with appropriate identification) is a monotone union of $M$. By arguments similar to Proposition 1, $M$ does not have the bounded union property.

Suppose $i_{\#}$ is an epimorphism. If $i_{\#}$ is also one-one, consider the double of $M, 2 M$. By Van Kampen's Theorem [6, p. 71], $\Pi_{1}(2 M)$ is isomorphic to $\Pi_{1}($ bdry $M)$. By [26, p. 305], this is impossible. Hence $i_{\#}$ is not a monomorphism and this case reduces to the first case.

The proof of the above theorem rests upon the fact that in both cases $M$ can be written in the form $M_{1} \# M_{2}$ where $M_{2}$ is a solid torus. In such a situation, $M$ is said to have a handle. We generalize this situation as follows. If $M$ is a compact 3-manifold such that $M=M_{1} \# M_{2}$ where $M_{2}$ is the closure of the complement of a polyhedral solid torus in the 3-sphere, $M$ is said to have a pseudohandle.

THEOREM 4. Let $M$ be a compact 3-manifold which has a pseudohandle. Then $M$ does not have the monotone union property. If the boundary of $M$ is connected and $M$ has a pseudohandle which is not a handle, then $M$ has the bounded union property.

We first prove this proposition for a special case.

Proposition 5. Suppose $M$ is the closure of the complement of a knotted solid torus $T$ in the 3-sphere $S^{3}$. If $g: M \rightarrow$ int $M$ is an embedding then either

(i) $M-g($ int $M$ ) is homeomorphic to (bdry $M) \times I$; or

(ii) $g(M)$ is contained in a 3-cell in $M$.

Proof. Let $T=\mathrm{Cl}\left(S^{3}-M\right)$ and $T^{\prime}=\mathrm{Cl}\left(S^{3}-g(M)\right)$. By Alexander [1], $T, T^{\prime}$ are solid tori. (For the terminology of this proof, see [22]. A summary of results in [22] appears in [9].) Let $t$ be a core (or center line) of $T$. There exist two possibilities.

Case 1. $t$ misses some meridial disk $D$ of $T^{\prime}$. Hence we may assume that $T$ misses some meridial disk of $T^{\prime}$; say $D$. Let $N$ be a regular neighborhood of $D \cup$ bdry $T^{\prime}$ in $T^{\prime}$ such that $N \cap T=\varnothing$. Then $g(M) \cup N$ is a three cell containing $g(M)$ and lying in $M$.

Case 2. $t$ meets each meridial disk of $T^{\prime}$. If $t$ is also a center line of $T^{\prime}$, then clearly $T^{\prime}-$ int $T$ is homeomorphic to (bdry $M$ ) $\times I$. Suppose $t$ is not a center line of $T^{\prime}$. Let $t^{\prime}$ be a center line of $T^{\prime}$. Then $t^{\prime}$ is a companion knot of $t$. Hence the genus of $t$ is strictly greater than the genus of $t^{\prime}$ [22, p. 192]. Therefore $g(M)$ and $M$ are not homeomorphic. This contradiction establishes the proposition. 
Proposition 6. Suppose $M$ is the closure of the complement of a knotted solid torus $T$ in $S^{3}$; then $M$ has the bounded union property. If $U=\bigcup U_{i}$ is a monotone union for $M, U$ is either homeomorphic to an open 3-cell or int $M$.

Proof. There are two cases to consider.

Case 1. For every positive integer $N$, suppose that there exists an integer $i=i(N)>N$ such that $U_{i-1}$ is contained in a 3-cell $C_{i}$ in $U_{i}$. Then

$$
U=\bigcup U_{i}=\bigcup_{N} C_{i(N)}
$$

is an open 3-cell.

Case 2. Suppose that there exists an integer $N$ such that for every $i>N, U_{i-1}$ is not contained in a 3-cell in $U_{i}$. By Proposition 5 , it follows that $U-U_{N}$ is homeomorphic to bdry $M \times(0,1)$ and hence $U$ is homeomorphic to int $M$.

PROPOSITION 7. Let $M$ be a compact 3-manifold which has a pseudohandle; say $M=M_{1} \# M_{2}$, where $M_{2}$ is the closure of the complement of a polyhedral solid torus in the 3-sphere. Let $U=\bigcup U_{i}$ be a monotone union of $M$. Then $U$ is homeomorphic to $M_{1} \#\left(\cup C_{i}\right)$ where $\bigcup C_{i}$ is a monotone union of $M_{2}$.

Proof. We can write $U_{1}=V_{0} \cup V_{1}$ such that $V_{0} \cap V_{1}=S$ is a 2-sphere missing the boundary of $U_{1}$ and such that $V_{0}$ is homeomorphic to the complement of an open 3-cell in $M_{1}$ and $V_{1}$ is homeomorphic to the complement of an open 3-cell in $M_{2}$. Consider any $U_{i}$. Then $U_{i}=V_{0} \cup V_{i}$ where $V_{0} \cap V_{i}=S$. From [15], $V_{i}$ is homeomorphic to the complement of an open 3-cell in $M_{2}$. Let $B$ be a 3-cell and let $C_{i}$ be formed from $V_{i} \cup B$ by identifying $S$ with the boundary of $B$. The proposition then follows.

Theorem 4 now follows from Propositions 5, 6, and 7. From Lemma 5 and Theorem 1 of [8], we can prove a converse of Theorem 4.

THEOREM 5. Let $M$ be a compact 3-manifold whose boundary is a torus. If $M$ has the bounded union property but not the monotone union property, then $M$ has a pseudohandle which is not a handle.

\section{The counterexample.}

THEOREM 6. There exists a compact 3-manifold $M$ such that the boundary of $M$ is a torus and $M$ has the monotone union property.

Construction of $M$. Let $P_{1}, P_{2}$ be solid tori. Let $c, a$ be longitudinal curves of $P_{1}$, $P_{2}$, respectively, and let $d, b$ be meridian curves of $P_{1}, P_{2}$, respectively, such that $c \cap d$ and $a \cap b$ are single points [22], [7, p. 2]; for example, see Figure 2. $S^{2} \times S^{1}$, the product of the 2-sphere with the 1-sphere, can be formed from $P_{1} \cup P_{2}$ by identifying their boundaries by a homeomorphism $h$ : bdry $P_{2} \rightarrow$ bdry $P_{1}$ such that the induced map $H: \pi_{1}\left(\right.$ bdry $\left.P_{2}\right) \rightarrow \pi_{1}\left(\right.$ bdry $\left.P_{1}\right)$ has the property that $H(a)=c$ and $H(b)=d[19]$. (We shall denote a homotopy class by one of its representatives if no ambiguity results.) 

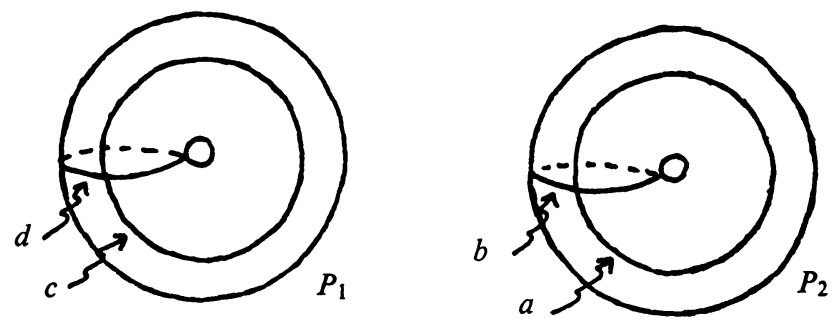

FIGURE 2

Let $\Gamma$ be the simple closed curve in int $P_{1}$ as indicated in Figure 3. Let $N$ be a regular neighborhood of $\Gamma$ in int $P_{1}$ and let $M=\left(S^{2} \times S^{1}\right)-$ int $N$.

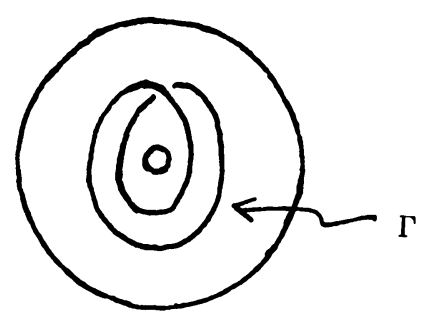

FIGURE 3

LEMMA 1. $\pi_{1}(M)=\left\{f, h: h \bar{f} \bar{h} \bar{f},(f h)^{2}(\bar{f} \bar{h})^{2}\right\}$.

Proof. Consider the link $\alpha$ given in Figure 4.

Then

$$
\begin{aligned}
\pi_{1}\left(S^{3}-\alpha\right) & =\pi_{1}\left(P_{1}-\Gamma\right) \\
& =\{e, f, g, h, i: h \bar{e} \bar{i} e, i \bar{f} \bar{h} f, g \bar{h} \bar{f} h, f \bar{h} \bar{e} h, f e \bar{f} \bar{g}\} \\
& =\{e, f, h, i: h \bar{e} \bar{i} e, i \bar{f} h f, f \bar{e} h, f e \bar{f} h \bar{f} h\} \\
& =\{e, f, h: e h \bar{e} \bar{f} h f, f \bar{h} \bar{e} h, f e \bar{f} h \bar{f} h\} \\
& =\left\{f, h:(f h)^{2}(\bar{f} \bar{h})^{2}\right\} .
\end{aligned}
$$

Note that $c$ is homotopic to $h$ and $d$ is homotopic to $\bar{e} \bar{f}=h \bar{f} h \bar{f}$. By Van Kampen's Theorem [6, p. 71], we have that

$$
\begin{aligned}
\pi_{1}(M) & =\left\{a, b, f, h: b, \bar{a} h, \bar{b} h \bar{f} \bar{h} \bar{f}, a b \bar{a} \bar{b},(f h)^{2}(\bar{f} \bar{h})^{2}\right\} \\
& =\left\{f, h: h \bar{f} \bar{h} \bar{f},(f h)^{2}(\bar{f} \bar{h})^{2}\right\} .
\end{aligned}
$$

LEMMA 2. $H_{1}(M)=Z \oplus Z_{2}$; i.e., the first homology group of $M$ is isomorphic to the direct sum of the integers and the integers mod 2.

Proof. Since $H_{1}(M)$ is obtained by abelianizing $\pi_{1}(M)$, we have that

$$
H_{1}(M)=\left\{f, h: f^{2}, f h \bar{f} \bar{h}\right\} .
$$

LeMma 3. Let $i$ : bdry $M \rightarrow M$ be the inclusion map. Then the induced map $i_{\#}: \pi_{1}($ bdry $M) \rightarrow \pi_{1}(M)$ is a monomorphism. 


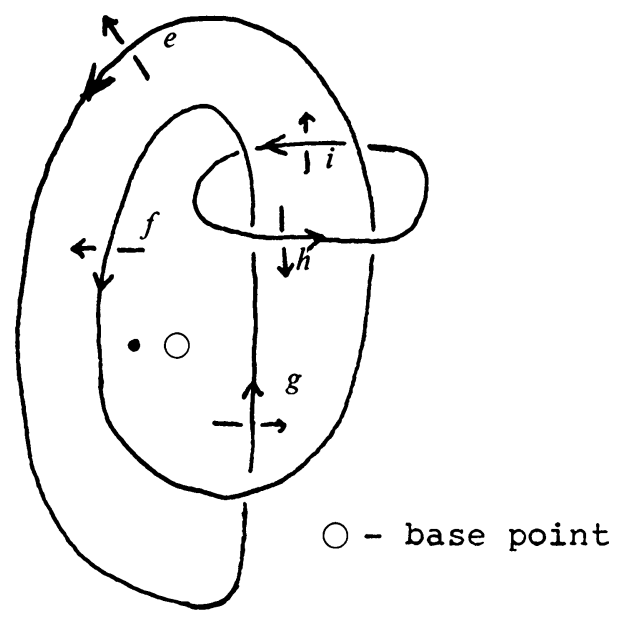

FIGURE 4

Proof. Suppose $i_{\#}$ is not one-one. From the remarks following Theorem 3, $M=M_{1} \# M_{2}$ where $M_{2}$ is a solid torus. Therefore $S^{2} \times S^{1}=M_{1} \#\left(M_{2} \cup N\right)$. By Milnor [15], $M_{2} \cup N$ is a 3-sphere. For, it follows from Van Kampen's Theorem that $M_{1}$ cannot be a 3-sphere. Then $C=\left(M_{2} \cup N\right) \cap\left(S^{2} \times S^{1}\right)$ is a closed 3-cell in $S^{2} \times S^{1}$ containing $\Gamma$. But $\Gamma$ is essential in $S^{2} \times S^{1}$, and therefore cannot lie in a 3-cell in $S^{2} \times S^{1}$. This contradiction establishes Lemma 3 .

LEMMA 4. $i_{\#} \pi_{1}($ bdry $M)=\left\{f, h^{2}: h^{2} \bar{f} \bar{h}^{2} f\right\}$.

Proof. From Figure 4, we obtain the presentation

$$
i_{\#} \pi_{1}(\text { bdry } M)=\{f, h f h: f(h f h) \bar{f}(\bar{h} \bar{f} \bar{h})\} .
$$

Note that $h f h=(\overline{f f}) h f h=\bar{f}(f h f) h=\bar{f}(h) h=\bar{f} h^{2}$ and the conclusion follows.

LEMMA 5. $i_{\#} \pi_{1}($ bdry $M)$ is a maximal subgroup of $\pi_{1}(M)$.

Proof. Note that the subgroup $\{f:\}$ generated by $f$ in $\pi_{1}(M)$ is normal in $\pi_{1}(M)$ for

$$
h f \bar{h}=(\bar{f} f) h f \bar{h}=\bar{f}(f h f) \bar{h}=\bar{f} h \bar{h}=\bar{f} .
$$

Define a homomorphism $\phi: \pi_{1}(M) \rightarrow\left\{h_{1}:\right\} \quad$ by $\phi(h)=h_{1}$ and $\phi(f)=1$. Then $\phi \mid i_{\#} \pi_{1}$ (bdry $\left.M\right): i_{\#} \pi_{1}$ (bdry $\left.M\right) \rightarrow\left\{h_{1}^{2}:\right\}$ is an epimorphism.

Note $\left\{h_{1}^{2}:\right\}$ is maximal in $\left\{h_{1}:\right\}$. Clearly $\{f:\} \subseteq i_{\#} \pi_{1}$ (bdry $\left.M\right)$. It follows from [24, pp. 140-141] that $i_{\#} \pi_{1}$ (bdry $M$ ) is maximal in $\pi_{1}(M)$.

Suppose $g: M \rightarrow$ int $M$ is an embedding. Let $R=M-\operatorname{int} g(M), T=g($ bdry $M)$.

LEMMA 6. $T$ bounds a solid torus in $S^{2} \times S^{1}$.

Proof. By Kinoshita [12, p. 791], there is a simple closed curve $S$ on $T$ such that $S$ does not bound a disk on $T$ but bounds a disk $D$ in $S^{2} \times S^{1}$ in such a way that $D \cap T=$ bdry $D=S$. Let $N$ be a regular neighborhood of $D$ in $S^{2} \times S^{1}$ which meets 
$T$ in a regular neighborhood of $S$ in $T$. Then $T^{\prime}=\mathrm{Cl}(T-N) \cup \mathrm{Cl}($ bdry $N-T)$ is a 2-sphere which, by Milnor [15], bounds a 3-cell $N^{\prime}$ in $S^{2} \times S^{1}$. Then $N \cup N^{\prime}$ is a solid torus which is bounded by $T$.

Lemma 7. The inclusion map $j_{T}: T \rightarrow R$ induces a monomorphism

$$
j_{T \#}: \pi_{1}(T) \rightarrow \pi_{1}(R) .
$$

Proof. Suppose to the contrary that $j_{T \#}$ is not a monomorphism. Then, as in the proof of Theorem 3, there is a 2-cell $D \subseteq R$ such that bdry $D=D \cap T$ is essential in $T$. Let $N^{\prime}$ be a regular neighborhood of $D$ in $R$ which meets $T$ in a regular neighborhood of bdry $D$ in $T[11, \mathrm{p} .735]$. Then

$$
\mathrm{Cl}\left(T-N^{\prime}\right) \cup \mathrm{Cl}\left(\text { bdry } N^{\prime}-T\right)
$$

is a 2 -sphere which bounds a 3 -cell in $S^{2} \times S^{1}$ containing $\Gamma$. This contradiction establishes the lemma.

Proof of Theorem 6. By Van Kampen's Theorem, $\pi_{1}(M)$ is the free product of $\pi_{1}(g M)=\pi_{1}(M)$ and $\pi_{1}(R)$ with amalgamated subgroup $i_{\#} \pi_{1}$ (bdry $\left.M\right)$. By Lemmas 3 and 7, the natural maps $\pi_{1}(T) \rightarrow \pi_{1}(g M)$ and $\pi_{1}(T) \rightarrow \pi_{1}(R)$ are monomorphisms. Hence by $[14$, p. 199], the inclusion map $j: R \rightarrow M$ induces a monomorphism $j_{\#}: \Pi_{1}(R) \rightarrow \Pi_{1}(M)$. Hence we have

$$
i_{\#} \pi_{1}(\text { bdry } M) \subseteq j_{\#} \pi_{1}(R) \subseteq \pi_{1}(M) .
$$

By Lemma 6, $R$ is embeddable in the 3-sphere and hence $H_{1}(R)$ has no element of finite order. Hence by Lemma $2, j_{\#} \pi_{1}(R) \neq \pi_{1}(M)$. Therefore, by Lemma 5 , $i_{\#} \pi_{1}($ bdry $M)=j_{\#} \pi_{1}(R)$; therefore the inclusion map $i^{\prime}:$ bdry $M \rightarrow R$ induces an isomorphism $i_{\#}^{\prime}: \pi_{1}$ (bdry $\left.M\right) \rightarrow \pi_{1}(R)$. Since bdry $M$ and $R$ are aspherical [21], bdry $M$ is a deformation retract of $R$ [4, p. 446]. Hence as argued on [4, p. 446], $R$ is homeomorphic to (bdry $M$ ) $\times I$. Hence $M$ has the concentricity property. By Theorem 1, $M$ has the monotone union property.

\section{REFERENCES}

1. J. W. Alexander, On the subdivision of 3-space by a polyhedron, Proc. Nat. Acad. Sci. U.S.A. 10 (1924), 6-8.

2. - The combinatorial theory of complexes, Ann. of Math. 31 (1930), 292-320.

3. R. H. Bing, An alternative proof that 3-manifolds can be triangulated, Ann. of Math. 69 (1959), 37-65.

4. E. Brown and R. Crowell, Deformation retractions of 3-manifolds into their boundaries, Ann. of Math. 82 (1965), 445-458.

5. M. Brown, The monotone union of open n-cells is an n-cell, Proc. Amer. Math. Soc. 12 (1961), 812-814.

6. R. Crowell and R. H. Fox, Introduction to knot theory, Ginn, Boston, Mass., 1963.

7. C. H. Edwards, Jr., Concentric solid tori in the 3-sphere, Trans. Amer. Math. Soc. 102 (1962), 1-17.

8. - Concentricity in 3-manifolds, Trans. Amer. Math. Soc. 113 (1964), 406-423. 
9. R. H. Fox, "A quick trip through knot theory" in Topology of 3-manifolds, edited by M. K. Fort, Jr., Prentice-Hall, Englewood Cliffs, N. J., 1962.

10. P. J. Hilton and S. Wylie, Homology theory, Cambridge Univ. Press, New York, 1962.

11. J. F. P. Hudson and E. C. Zeeman, On regular neighborhoods, Proc. London Math. Soc. (3) 14 (1964), 719-745.

12. S. Kinoshita, On Fox's property of a surface in a 3-manifold, Duke Math. J. 33 (1966), 791-794.

13. K. W. Kwun, Open manifolds with monotone union property, Proc. Amer. Math. Soc. 17 (1966), 1091-1093.

14. W. Magnus, A. Karass and D. Solitar, Combinatorial group theory, Interscience, New York, 1966.

15. J. Milnor, A unique decomposition theorem for 3-manifolds, Amer. J. Math. 84 (1962), $1-7$.

16. E. E. Moise, Affine structures in 3-manifolds. V, Ann. of Math. 56 (1952), 96-114.

17. M. H. A. Newman, On the superposition of n-dimensional manifolds, J. London Math. Soc. 2 (1927), 56-64.

18. M. H. A. Newman and J. H. C. Whitehead, On the group of a certain linkage, Quart. J. Math. Oxford Ser. (2) 8 (1937), 14-21.

19. J. Nielsen, Untersuchungern zur Topologie der geschlossen zweiseitigen Flächen, Acta Math. 50 (1927), 266.

20. C. D. Papakyriakopoulos, On solid tori, Proc. London Math. Soc. (3) 7 (1957), 281-299.

21. - On Dehn's lemma and the asphericity of knots, Ann. of Math. 66 (1957), 1-26.

22. H. Schubert, Knoten und Vollringe, Acta Math. 90 (1953), 131-286.

23. E. H. Spanier, Algebraic topology, McGraw-Hill, New York, 1966.

24. B. L. van der Waerden, Modern algebra, Ungar, New York, 1953.

25. J. H. C. Whitehead, A certain open manifold whose group is unity, Quart. J. Math. Oxford Ser. (2) 6 (1935), 364-366.

26. H. Hopf, Fundamentalgruppe und zweite Bettische Gruppe, Comment. Math. Helv. 14 (1942), 257-309.

The Florida State University,

Tallahassee, Florida 